\title{
Full Phase Coverage of BY Dra with the VLA
}

\author{
Jean-Pierre Caillault ${ }^{1}$ and Stephen Drake ${ }^{2}$ \\ ${ }^{1}$ University of Georgia, Athens, GA 30602 USA \\ ${ }^{2}$ SMM Data Analysis Center, Greenbelt, MD 20770 USA
}

\begin{abstract}
We have obtained 21 one-hour observations of the prototypical spotted star, BY Dra, with the VLA. These data are sufficiently well spaced in time to allow for complete rotational and orbital phase coverage; the resultant radio light curves are compared to their optical counterpart. We also plot the emission versus time without phase-folding, in order to search for longer periods of activity. Splitting the data into smaller (three minute) time bins allows for the determination of the normalized amplitude distribution of the flux; we compare this with known stellar optical and X-ray flare distributions.
\end{abstract}

\section{Observations}

We observed BY Dra with the VLA in April 1988. Twenty-one one-hour integrations were made at a wavelength of $6 \mathrm{~cm}$. We show these data in Fig. 1. Although it is possible that a general decline in the level of emission occurred over the course of the month, it is likely that random large flaring was responsible for the higher fluxes detected; in fact, a large flare (peak flux $\sim 6.5 \mathrm{mJy}$ ) was detected on 6 April. We never failed to detect BY Dra (at the $3 \sigma$ level), with the lowest one-hour integrated flux being $\sim 0.30 \mathrm{mJy}$. The degree of polarization is generally small; hence, we interpret this low-level flux as quiescent emission from BY Dra.

\section{Low-level flaring}

Each of the 21 one-hour observations was broken up into three-minute "snapshots". By defining a quantity $F_{r}$ as the ratio of the flux in any one snapshot to the minimum snapshot flux observed $(\sim 0.20 \mathrm{mJy})$, we have constructed a histogram $N\left(F_{r}\right)$ of the normalized amplitude distribution of three-minute snapshot fluxes of BY Dra; this is presented in Fig. 2. This distribution is almost flat, differing greatly from that found by Kunkel (1973) for $U$-band flares in dMe stars, by Drake (1971) for solar X-ray flares, by Montmerle et al. (1983) for the $\rho$ Oph X-ray sources, and by Caillault and Zoonematkermani (1990) for the Orion X-ray sources (all roughly described by a power-law with exponent $\alpha \sim-1.4 \pm 0.2$ ). Hence, the quiescent emission does not seem to result from low-level flaring. 


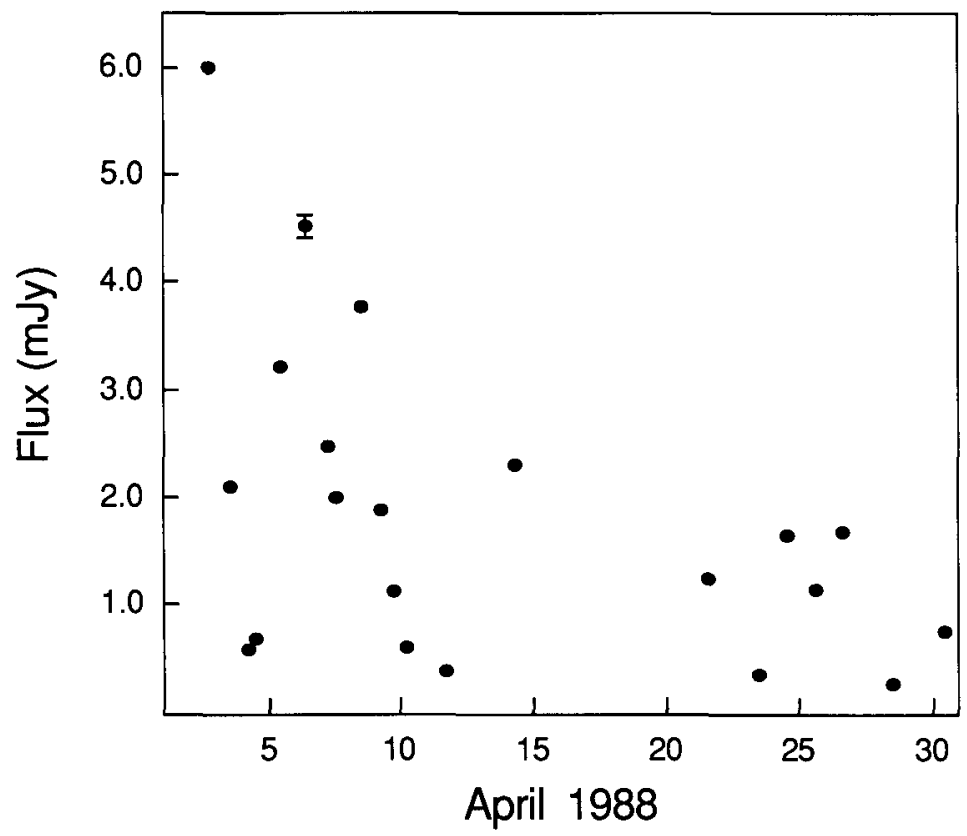

Fig. 1. The 21 one-hour integrations of BY Dra taken during April 1988 are plotted as filled circles. A representative error bar for these data is shown on the point corresponding to April 6 ( $\sim 4.5 \mathrm{mJy}$ ). (This point is also the "flare" which is mentioned in the text.)

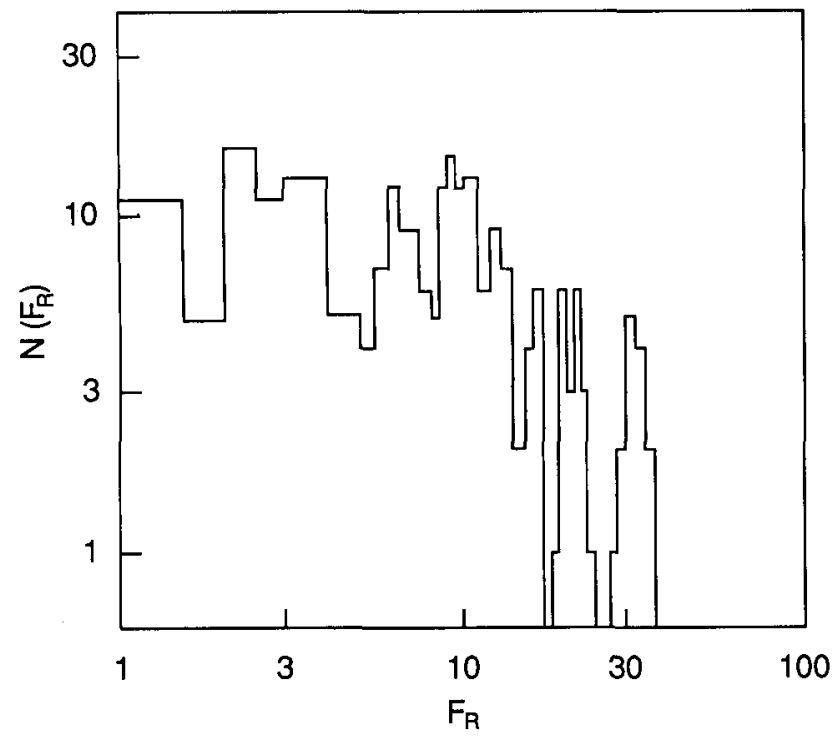

Fig. 2. A histogram $N\left(F_{r}\right)$ of the normalized amplitude distribution of three-minute "snapshot" fluxes of BY Dra. 


\section{Rotational modulation}

Rotational modulation of the flux is a way of determining the size and location of the quiescent source (Gary, 1985). This has been attempted by Linsky and Gary (1983) and Gary (1986) for the eclipsing binary YY Gem and by Caillault et al. (1989) for the white-dwarf, K-dwarf eclipsing binary V471 Tau. The VLA data obtained in the present study are sufficiently well spaced in time to allow for complete rotational ( $P=3.83^{d}$ ) phase coverage of BY Dra; the resultant radio light curve is shown in Fig. 3 (this figure also includes data from 1986, Caillault et al., 1988). An anti-correlation of the radio emission with BY Dra's optical emission (Cutispoto et al., 1987) would provide direct evidence of the presumed magnetic origin of the $6 \mathrm{~cm}$ emission; no such pattern is seen). (We have also plotted the emission versus the orbital $\left[P=5.98^{d}\right]$ phase; again, no pattern is seen). The optical observations were performed in 1986, though; simultaneous, or even contemporaneous, data would have been better suited to this study. As it turns out, the inclination angle of BY Dra is $\sim 30^{\circ}$ and the amplitude of optical variation is $\leqq 0.1 \mathrm{mag}$, requiring a very meticulous experiment be performed even without the complication provided by the random large flaring. In addition, if the radio emission results from a coronal gyrosynchrotron mechanism, then large loops might preclude any rotational modulation.

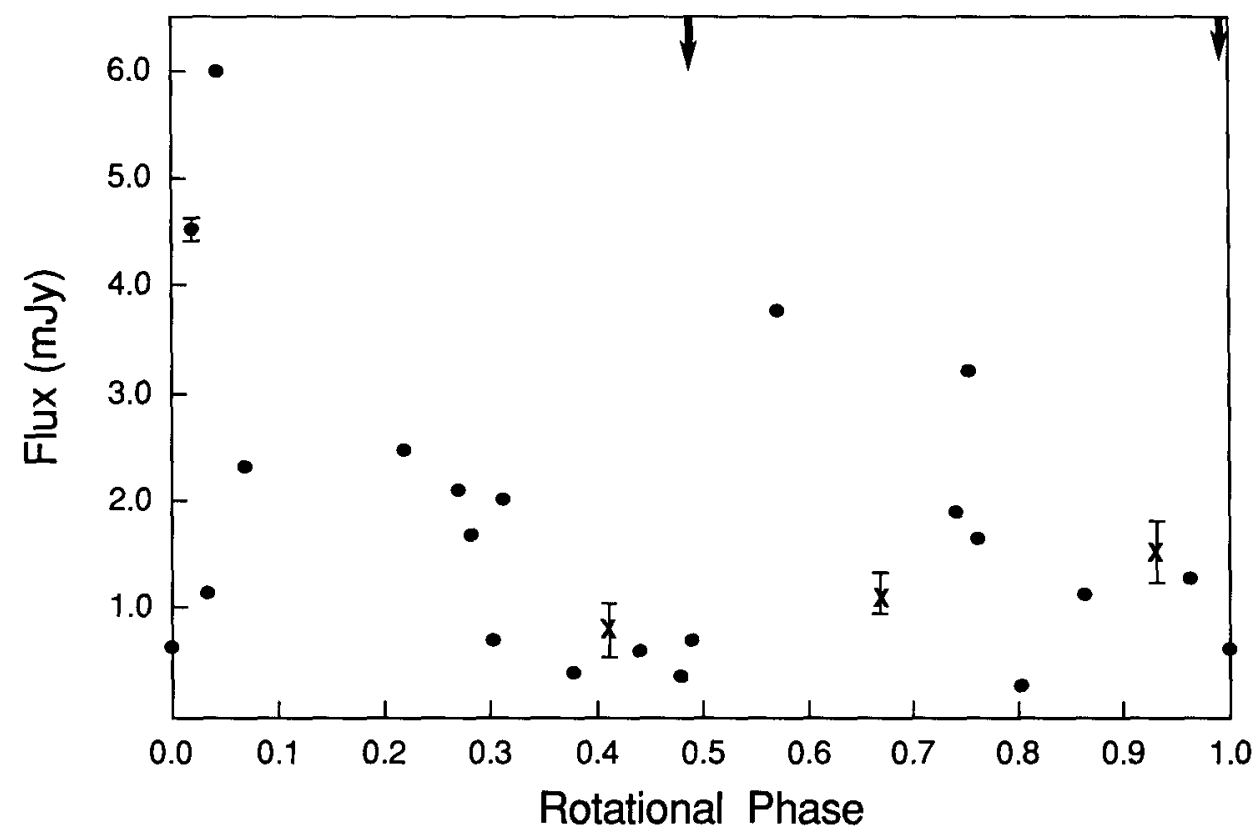

Fig. 3. The same data as in Fig. 1, but with the addition of crosses representing data obtained in February 1986. The optical light maximum and minimum represented by the two arrows at the top of the figure, are at $\phi=0.49$ and $\phi=0.99$, respectively. 


\section{References}

Caillault, J.-P., Drake, S., Florkowski, D.: 1988, Astron. J. 95, 887

Caillault, J.-P., Patterson, J.., Skillman, D.: 1989, in IAU Colloquium No 104 Solar and Stellar Flares, eds. B.M. Haisch and M. Rodonò, Catania p. 119

Caillault, J.-P., Zoonematkermani, S.: 1990, in Flare Stars in Star Clusters, Associations and in the Solar Vicinity, eds. L.V. Mirzoyan et al., Kluwer p. 159

Cutispoto, G., Leto, G., Pagano, I., Santagati, G., Ventura, R.: 1987, Inf. Bull. Var. Stars, No 3102

Drake, J.F.: 1971, Solar Physics 16, 152

Gary, D.E.: 1985, in Radio Stars, eds. R. Hjellming, D.M. Gibson, Reidel, Dordrect, p. 185

Gary, D.E.: 1986, in Fourth Cambridge Workshop on Cool Stars, Stellar Systems, and the Sun, eds. M. Zeilik and D.M. Gibson, Springer, Berlin, p. 235

Kunkel, W.E.: 1973, Astrophys. J. Suppl. 25, 1

Linsky, J.L., Gary, D.E.: 1983, Astrophys. J. 274, 776

Montmerle, T., Koch-Miramond, L., Falgarone, E., Grindlay, J.E.: 1983, Astrophys. J. 269, 182 